\title{
Fast Implementation of a General $L / M$ Rate Changer by a Filter Bank Structure
}

\author{
Wing-kuen Ling and P. K. S. Tam \\ Department of Electronic and Information Engineering \\ The Hong Kong Polytechnic University \\ Hung Hom, Kowloon, Hong Kong \\ Hong Kong Special Administrative Region, China \\ Tel: (852) 2766-6238, Fax: (852) 2362-8439 \\ Email: bingo@encserver.eie.polyu.edu.hk, enptam@polyu.edu.hk
}

\begin{abstract}
In this paper, we show that an $L / M$ rate changer can be realized as a discrete time SISO $(L, M)$ shift invariant system in form of a two-dimensional kernel function or a filter bank structure. Based on this realization, we can implement an $L / M$ rate changer by a bank of filters with the average number of the coefficients in the filters in each channel is $1 / L$ of the original $L / M$ rate changer. Hence, the system is speed up by $L$. This helps the designer to design a sharp cutoff discrete time FIR filters in an $L / M$ rate changer for some real time applications in video systems.
\end{abstract}

Index Terms $-L / M$ rate changer, discrete time SISO linear $(L, M)$ shift invariant system, kernel function, filter bank structure, sharp cutoff discrete time FIR filters

\section{INTRODUCTION}

An $L / M$ rate changer shown in figure 1 plays an important role in the audio, image and video systems [1]. A good $L / M$ rate changer sometimes requires a sharp cutoff discrete time FIR filter $h[n]$, especially those applied in the digital image and the digital video systems. However, a sharp cutoff discrete time FIR filter always contains a lot of coefficients. As a result, it requires a very long processing time and restricts in some of the real time applications in video systems.

In this paper, a parallel processing technique is proposed to break down a single $L / M$ rate changer into a multi-channel system shown in figure 2 , such that the average number of coefficients of the filters in each channel $h_{j}[n]$, for $j=0,1, \ldots, L-1$, is $1 / L$ that of the original $L / M$ rate changer. This will speed up the system by $L$.

\section{REALIZATION OF AN $L / M$ RATE CHANGER AS A DISCRETE TIME SISO LINEAR $(L, M)$ SHIFT INVARIANT SYSTEM}

When the input of an $L / M$ rate changer shifts by $M$ samples, the output will shift by $L$ samples. Hence, an $L / M$ rate changer is a discrete time SISO linear $(L, M)$ shift invariant system. As a discrete time SISO linear $(L, M)$ shift invariant system can be characterized by a two-dimensional kernel function $g[n, k]$, where $g[n, k]=g[n-L, k-M], \forall k, n \in \mathbf{Z}$ [2], or by a filter bank structure shown in figure 2 [3], so we can realize an $L / M$ rate changer $h[n]$ in terms of a two-dimensional kernel function or a filter bank structure. The transformation from an $L / M$ rate changer to a discrete time SISO linear $(L, M)$ shift invariant system is summarized in the following two theorems:

\section{Theorem 1}

Given an $L / M$ rate changer shown in figure 1 , there exists a discrete time SISO linear $(L, M)$ shift invariant system, characterized by a two-dimensional kernel function $g[n, k]$ with $g[n, k]=h[n \cdot M-k \cdot L], \forall k, n \in \mathbf{Z}$, such that the input output relationship of the discrete time SISO linear $(L, M)$ shift invariant system will be exactly the same as that of an $L / M$ rate changer. The proof is as follows:

The input output relationship of the discrete time SISO linear $(L, M)$ shift invariant system is governed by [2]:

$y[n]=\sum_{k \rightarrow-\infty}^{+\infty} g[n, k] \cdot x[k], \quad \forall n \in Z$

Let $g[n, k]=h[n \cdot M-k \cdot L], \forall k, n \in \mathbf{Z}$, we have:

$y[n]=\sum_{k \rightarrow-\infty}^{+\infty} h[n \cdot M-k \cdot L] \cdot x[k], \quad \forall n \in Z$

which is the same input output relationship of the $L / M$ rate changer [1], so this proves the theorem.

For example, if the upsampler and the downsampler of an $L M$ rate changer is 2 and 3 respectively, and the filter is:

$h[n]=\left\{\begin{array}{cc}\frac{1}{2} \cdot \sin c\left(\frac{n}{2}\right) \quad ; \text { for } & -10 \leq n \leq 10, \\ 0 \quad ; & \text { otherwise }\end{array}\right.$

then the two-dimensional kernel function of the discrete time SISO linear $(L, M)$ shift invariant system is:

$g[0, k]=0.5 \cdot \delta[k]$,

$g[1, k]=\left\{\begin{array}{cc}\frac{1}{2} \cdot \sin c\left(\frac{3-2 \cdot k}{2}\right) & ; \text { for }-3 \leq k \leq 6, \\ 0 & ; \text { otherwise }\end{array}\right.$

and $g[n, k]=g[n-2, k-3]$ for other integer values of $n$.

Theorem 2

Given an $L / M$ rate changer shown in figure 1 , there 
exists a discrete time SISO linear $(L, M)$ shift invariant system, characterized by a filter bank structure shown in figure 2 with $h_{j}[n]=h[j \cdot M+n \cdot L]$, for $\forall n \in \mathbf{Z}$ and $j=0,1, \ldots, L-1$, such that the input output relationship of the discrete time SISO linear $(L, M)$ shift invariant system will be exactly the same as that of an $L / M$ rate changer. The proof is as follows:

The input output relationship of the discrete time SISO linear $(L, M)$ shift invariant system is governed by:

$$
y[n]=\left\{\begin{array}{cc}
\sum_{k \rightarrow-\infty}^{+\infty} x[k] \cdot h_{0}\left[\frac{n \cdot M}{L}-k\right] & ; \text { if } \bmod (n, L)=0 \\
\sum_{k \rightarrow-\infty}^{+\infty} x[k] \cdot h_{1}\left[\frac{(n-1) \cdot M}{L}-k\right] & ; \text { if } \bmod (n, L)=1 \\
\vdots & \vdots \\
\sum_{k \rightarrow-\infty}^{+\infty} x[k] \cdot h_{L-1}\left[\frac{(n-L+1) \cdot M}{L}-k\right] & ; \text { if } \bmod (n, L)=L-1
\end{array}\right.
$$

Let $h_{j}[n]=h[j \cdot M+n \cdot L]$, for $\forall n \in \mathbf{Z}$ and $j=0,1, \ldots, L-1$, we have:

$$
y[n]=\sum_{k \rightarrow-\infty}^{+\infty} h[n \cdot M-k \cdot L] \cdot x[k], \quad \forall n \in Z
$$

which is also the same input output relationship of the $L / M$ rate changer, hence, the theorem is proved.

For the same example described after theorem 1, the filters of the filter bank structure of the discrete time SISO linear $(L, M)$ shift invariant system are:

$h_{0}[n]=0.5 \cdot \delta[n]$ and

$$
h_{1}[n]=\left\{\begin{array}{cc}
\frac{1}{2} \cdot \sin c\left(\frac{3 \cdot n+2}{2}\right) & ; \text { for }-6 \leq n \leq 3 \\
0 & ; \text { otherwise }
\end{array}\right.
$$

\section{REALIZATION OF A DISCRETE TIME SISO LINEAR $(L, M)$ SHIFT INVARIANT SYSTEM AS AN $L / M$ RATE CHANGER}

It can been shown that an $L / M$ rate changer is input output equivalent to a discrete time SISO linear $(L, M)$ shift invariant system if and only if $L$ and $M$ is co-prime. Hence, only the discrete time SISO linear $(L, M)$ shift invariant system with $L$ and $M$ is co-prime can be realized as an $L / M$ rate changer. Under this condition, the transformation from a discrete time SISO linear $(L, M)$ shift invariant system to an $L / M$ rate changer is summarized in the following two theorems:

\section{Theorem 3}

Given a discrete time SISO linear $(L, M)$ shift invariant system with $L$ and $M$ is co-prime, there exists an $L / M$ rate changer shown in figure 1 with $h[n \cdot M-k \cdot L]=g[n, k], \forall n, k \in \mathbf{Z}$, such that the input output relationship of the $L M$ rate changer will be exactly the same as that of the discrete time SISO linear $(L, M)$ shift invariant system. The proof is as follows:

The input output relationship of the $L / M$ rate changer is governed by [1]:

$$
y[n]=\sum_{k \rightarrow-\infty}^{+\infty} h[n \cdot M-k \cdot L] \cdot x[k], \quad \forall n \in Z
$$

Since $h[n \cdot M-k \cdot L]=g[n, k], \forall n, k \in \mathbf{Z}$, we have: $y[n]=\sum_{k \rightarrow-\infty}^{+\infty} g[n, k] \cdot x[k], \quad \forall n \in Z$

which is the same input output relationship of the discrete time SISO linear $(L, M)$ shift invariant system, so this proves the theorem.

For example, if $L$ and $M$ of a discrete time SISO linear $(L, M)$ shift invariant system are 2 and 3 respectively, and the two-dimensional kernel function is:

$g[0, k]=k, g[1, k]=2 \cdot k$ and $g[n, k]=g[n-2, k-3]$ for other integer values of $n$, then the filter in an $L / M$ rate changer is:

$$
h[n]=\left\{\begin{array}{cc}
-\frac{n}{2} & ; \text { for } \mathrm{n} \text { is even, } \\
3-n & ; \text { for } \mathrm{n} \text { is odd }
\end{array}\right.
$$

\section{Theorem 4}

Given a discrete time SISO linear $(L, M)$ shift invariant system with $L$ and $M$ is co-prime, there exists an $L / M$ rate changer shown in figure 1 with $h[j \cdot M+n \cdot L]=h_{j}[n]$, for $\forall n \in \mathbf{Z}$ and $j=0,1, \ldots, L-1$, such that the input output relationship of the $L / M$ rate changer will be exactly the same as that of the discrete time SISO linear $(L, M)$ shift invariant system. The proof is as follows:

The input output relationship of the $L / M$ rate changer is governed by [1]:

$$
y[n]=\sum_{k \rightarrow-\infty}^{+\infty} h[n \cdot M-k \cdot L] \cdot x[k], \quad \forall n \in Z
$$

Since $h[j \cdot M+n \cdot L]=h_{j}[n]$, for $\forall n \in \mathbf{Z}$ and $j=0,1, \ldots, L-1$, we have:

$$
y[n]=\left\{\begin{array}{cc}
\sum_{k \rightarrow-\infty}^{+\infty} x[k] \cdot h_{0}\left[\frac{n \cdot M}{L}-k\right] & ; \text { if } \bmod (n, L)=0 \\
\sum_{k \rightarrow-\infty}^{+\infty} x[k] \cdot h_{1}\left[\frac{(n-1) \cdot M}{L}-k\right] & ; \text { if } \bmod (n, L)=1, \quad(12), \\
\vdots & \vdots \\
\sum_{k \rightarrow-\infty}^{+\infty} x[k] \cdot h_{L-1}\left[\frac{(n-L+1) \cdot M}{L}-k\right] & ; \text { if } \bmod (n, L)=L-1
\end{array}\right.
$$

which is also the same input output relationship of the discrete time SISO linear $(L, M)$ shift invariant system, hence, the theorem is proved.

For example, if the upsampler and the downsampler of the discrete time SISO linear $(L, M)$ shift invariant system are 2 and 3 respectively, and the filters of the filter bank structure are $h_{0}[n]=n$ and $h_{l}[n]=2 \cdot n$ respectively, then the filter in an $L / M$ rate changer is:

$$
h[n]=\left\{\begin{array}{cc}
\frac{n}{2} & ; \text { for } \mathrm{n} \text { is even, } \\
n-3 & ; \text { for } \mathrm{n} \text { is odd }
\end{array}\right.
$$

\section{APPLICATION ON FAST IMPLEMENTATION OF AN $L / M$ RATE CHNAGER}

By realizing an $L / M$ rate changer as a discrete time SISO linear $(L, M)$ shift invariant system in form of a filter bank structure shown in figure 2, the average number of coefficients of the filters in each channel $h_{j}[n]$, for $j=0,1, \ldots, L-1$, is $1 / L$ that of the original $L M$ rate changer, this will speed up the system by $L$. 
The transformation helps the user to design an $L / M$ rate changer with a sharp cutoff discrete time FIR filter $h[n]$, and can be applied in real time applications in video systems.

\section{CONCLUSION}

In this paper, we show that a discrete time $\operatorname{SISO}(L, M)$ shift invariant system with $L$ and $M$ is co-prime can be realized as an $L / M$ rate changer. Also, we show that an $L / M$ rate changer can be realized as a discrete time SISO $(L, M)$ shift invariant system in form of a two-dimensional kernel function or a filter bank structure shown in figure 2. Based on this realization, we can implement an $L / M$ rate changer by a bank of filters with the average number of the coefficients in the filters in each channel is $1 / L$ of the original $L / M$ rate changer. This can speed up the system by $L$ and helps the designer to design a sharp cutoff discrete time FIR filters in an $L / M$ rate changer for some real time applications in video systems.

\section{ACKNOWLEDGEMENT}

The work described in this letter was substantially supported by a grant from the Hong Kong Polytechnic University with account number G-V968.

\section{REFERENCES}

[1] P. P. Vaidyanathan, Multirate Systems and Filter Banks, Englewood Cliffs, NJ: Prentice-Hall, 1993.

[2] Tongwen Chen, Li Qiu and Er-Wei Bai, "Generate Multirate Building Structures with Application to Nonuniform Filter Banks," IEEE Transaction on Circuits and Systems-II: Analog and Digital Signal Processing, vol. 45, No. 8, pp. 948-958, August, 1998.

[3] Wing-kuen Ling and Kwong-Shun Tam, "Theory of Discrete Time SISO Linear $(L, M)$ Shift Invariant System”, IEEE International Symposium on Intelligent Multimedia, Video and Speech Processing, May, 2001.
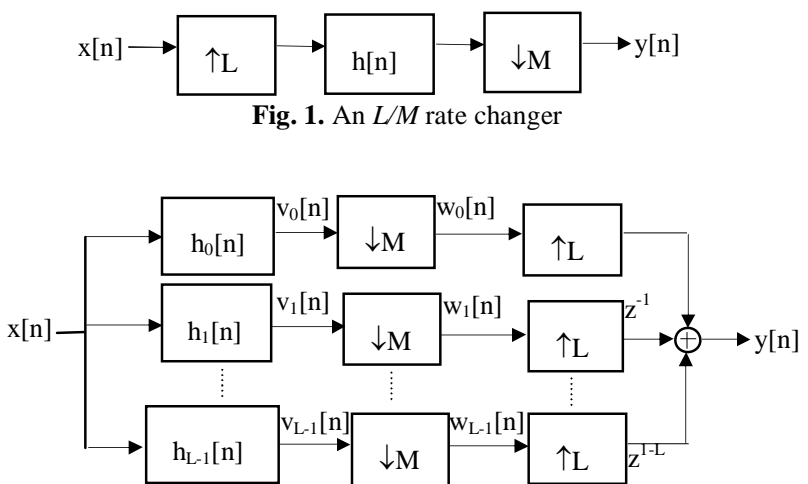

Fig. 2. Filter bank realization of an $L / M$ rate changer 